\title{
Tomographie ultrasonore pour les arbres sur pied
}

\author{
Roberto MARTINIS ${ }^{\mathrm{a} *}$, Laura Valentina SOCCO $^{\mathrm{b}}$, Luigi SAMBUELLI $^{\mathrm{b}}$, Giovanni NiCOLOTTI ${ }^{\mathrm{a}}$, \\ Olivier SCHMITT ${ }^{\mathrm{c}}$, Voichita BUCUR ${ }^{\mathrm{d}}$ \\ a Università degli Studi di Torino, DI.VA.P.R.A via Leonardo da Vinci 44, 10095 Grugliasco (TO), Italy \\ b Politecnico di Torino, DIGET, C.so Duca degli Abruzzi, 24, 10129 Torino, Italy \\ ${ }^{c}$ Direction de Parcs et Jardins de la ville de Nancy, Place Stanislas, 54000 Nancy, France \\ d INRA Centre de Nancy, Lermab, UMR 1093, 54280 Champenoux, France
}

(Received 1 October 2002; accepted 22 January 2003)

\begin{abstract}
Résumé - La tomographie par ultrasons a été utilisée pour détecter l'état de dégradation de la section transversale d'un tronc de hêtre, attaqué par une pourriture blanche. La reconstitution de l'image du tronc en 2D a été faite à partir d'un logiciel qui utilise les valeurs de vitesses de propagation des ultrasons à travers la section de l'arbre. Le bois dégradé est caractérisé par des valeurs faibles des vitesses. Les mesures ont été effectuées à travers l'écorce de l'arbre, à trois hauteurs du sol, avec des transducteurs spéciaux de fréquence d'émission de $1 \mathrm{MHz}$. Dans le cas d'un signal trop entaché par le bruit, une analyse en fréquence a été effectuée. Les tomogrammes ultrasonores ainsi obtenus ont été comparés avec les photographies des sections correspondantes et avec les données obtenues par mesure de résistance à la pénétration d'une aiguille, avec un «Résistograph ». Aux faibles valeurs de la résistance à la pénétration correspondent des valeurs faibles de vitesses. Une bonne correspondance entre les tomogrammes et les photographies a été constatée. La résolution de l'image tomographique est comprise entre 4 et $5 \mathrm{~cm}$. À l'état actuel du développement des techniques de détection non destructives acoustiques des défauts sur les arbres sur pied, cette résolution est la meilleure jamais obtenue.
\end{abstract}

\section{détection non destructive / tomographie ultrasonore / pourriture du bois / évaluation de la stabilité des arbres}

\begin{abstract}
Ultrasonic tomography on standing trees. Ultrasonic tomography was used for the detection of degradation of transversal section of a beech, attacked by white decay. The image reconstruction of the transversal section of the tree in 2D was obtained with a software, which used the values of ultrasonic velocities measured at different heights from the ground. The attacked wood is characterised by low velocities. The measurements were performed with special transducers of $1 \mathrm{MHz}$, without damaging the bark of the tree. To improve the readings of the signals, a frequency analysis was performed. The ultrasonic tomographies were compared with the corresponding photographic images and with data obtained with a "Resistograph". A good agreement between ultrasonic tomographies and photographs was observed. The image resolution is between 4 and $5 \mathrm{~cm}$. Presently, this is the best resolution ever obtained with an acoustic nondestructive method on a standing tree.
\end{abstract}

non invasive wood testing / ultrasonic tomography / wood decay / tree stability assessment

\section{INTRODUCTION}

La stabilité mécanique des arbres sur pied des parcs et jardins publiques est déterminée, entre autres, par le degré de détérioration de la structure transversale du tronc. Couramment la pathologie du tronc est déterminée par différentes techniques comme par exemple : le prélèvement des carottes de sondage, le test mécanique de dureté qui consiste à mesurer la résistance du bois à la pénétration d'un outil de type aiguille, avec un appareil dénommé « Résistograph », ou le test acoustique qui détermine la réponse de l'arbre à une onde de choc [6, 12-14, 17].

Récemment, pour examiner la section transversale d'un arbre sur pied plusieurs techniques non destructives ont été développées, comme par exemple :

- la tomographie par rayons X [7, 8] qui a l'avantage d'une bonne résolution de l'image, pouvant aller jusqu'à $5 \mu \mathrm{m}$ [4], mais qui utilise une source radioactive ;
- la tomographie par résonance magnétique [10] qui est très fiable mais difficilement applicable in situ ;

- le radar [9] qui est une technique très rapide mais qui nécessite de la part de l'opérateur une grande expérience pour l'interprétation de l'image ;

- l'imagerie par ultrasons [2,15] qui dans l'état actuel du développement de la technologie nécessite le contact direct des transducteurs avec l'objet à inspecter (tronc de l'arbre, poteau, etc.).

Le but de cette recherche est de démontrer la fiabilité de la tomographie par ultrasons, par mesure du temps de propagation des impulsions ultrasonores, pour détecter l'état de dégradation de la section transversale d'un arbre sur pied, en utilisant un appareil portable et un logiciel original pour la reconstitution de l'image en 2D et en 3D. Les tomogrammes des vitesses de propagation des ultrasons ainsi obtenus seront comparés, d'une

* Corresponding author: roberto.martinis@unito.it 


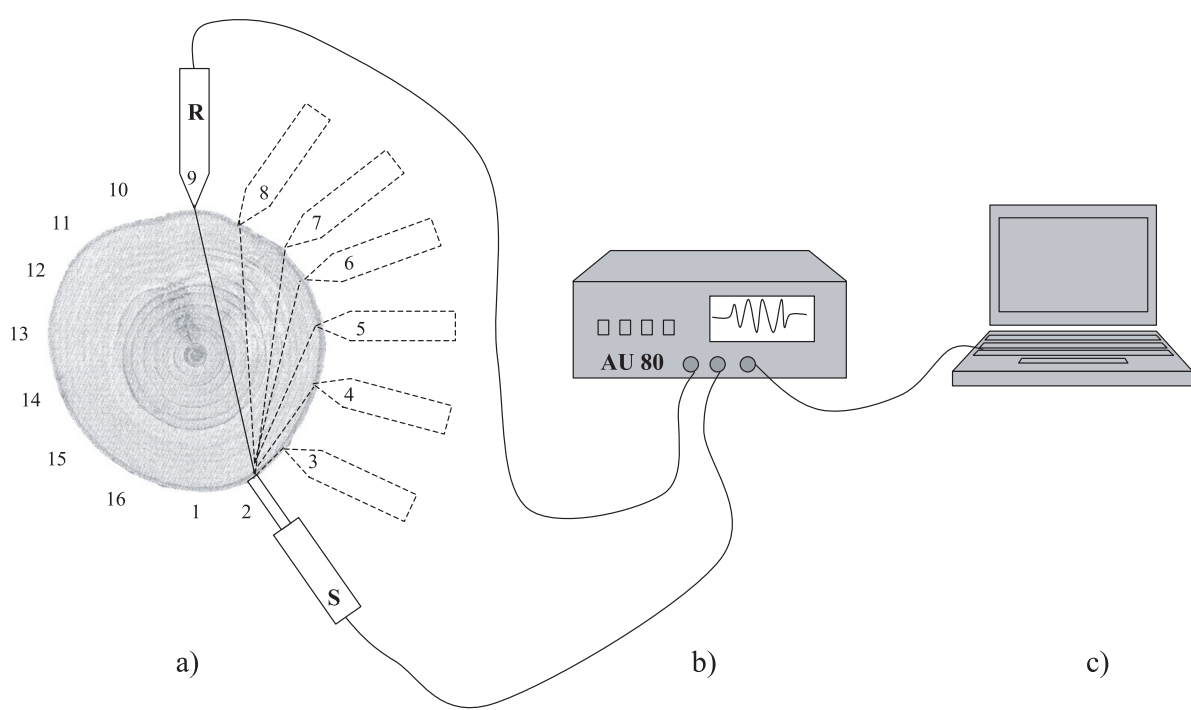

Figure 1. Schéma d'acquisition des données: (a) mesures sur la section d'un arbre ; (b) lecture des temps ; (c) analyse du signal. $\mathrm{S}$ : transducteur source $; \mathrm{R}$ : transducteur récepteur. part avec les photographies des différentes sections du tronc après abattage et d'autre part, avec les résultats de la méthode de la pénétration d'une aiguille de l'appareil connu sous le nom de « Résistograph».

\section{MATÉRIELS ET METHODES}

\subsection{Matériels}

La tomographie ultrasonore a été effectuée sur la section transversale d'un hêtre sur pied (Fagus sylvatica) situé au parc Sainte Marie à Nancy, d'un diamètre $60 \mathrm{~cm}$ et d'une hauteur $15 \mathrm{~m}$, qui présentait une dégradation (pourriture blanche) du tronc bien visible. Par ailleurs le houppier de cet arbre était encore bien développé et ne semblait pas être affecté par la dégradation du tronc. Le Service des Espaces Verts, des Parcs et Jardins de la ville de Nancy était intéressé de connaître l'état de dégradation du tronc de cet arbre pour décider en toute objectivité de son abattage.

\subsection{Méthodes}

La tomographie par ultrasons reconstitue l'image de l'objet analysé à partir de l'étude de la distribution des valeurs des vitesses des impulsions ultrasonores qui traversent l'objet. Cette technique est couramment utilisée en Géophysique. Pour le matériau bois attaqué par des agents fongiques, la diminution des valeurs des vitesses reflète cette dégradation. Les champignons, en effet, dégradent tous les composants de la paroi des cellules, et réduisent, outre la masse, les propriétés élastiques du bois et donc la vitesse de propagation des ultrasons $[3,11]$.

La section transversale de l'arbre a été étudiée à trois niveaux, à $36 \mathrm{~cm}$ du sol (section A), $84 \mathrm{~cm}$ (section B) et $138 \mathrm{~cm}$ (section C) par tomographie ultrasonore et par mesure avec le «Résistograph». A chaque niveau la circonférence de l'arbre a été divisée en 16 zones pour obtenir finalement 16 points de mesure du temps de propagation d'une impulsion ultrasonore. Chaque point de mesure a ensuite servi d'une part, comme repère de positionnement pour la source d'émission ultrasonore et d'autre part, pour la réception du signal, constituant ainsi un réseau qui couvre toute la section du tronc selon la méthodologie proposée par Martinis [9] (Fig. 1). D'abord on a placé le transducteur source sur le point 1 et le récepteur sur les autres 15 points. Ensuite le transducteur source a été placé sur le point 2 et le récepteur sur les points allant de 3 à 16. On a poursuivi l'opération jusqu'au positionnement de la source sur le point 16 et du récepteur sur le point 15 . En changeant la position des transducteurs source et de réception on a obtenu 120 lectures indépendantes de temps de première arrivée. Les coordonnées de chaque point de mesure ont été mesurées pour la reconstitution de l'image tomographique. Les temps et les coordonnées ont permis de procéder à l'inversion tomographique et d'obtenir l'image de la distribution des vitesses dans la section.

Les transducteurs ont été positionnés sur la périphérie du tronc, et les mesures ont été effectuées à travers l'écorce de l'arbre. L'intégrité de l'écorce de l'arbre n'a nullement été affectée par les mesures ultrasonores.

L'image ultrasonore de chaque section a été reconstituée à partir de 120 mesures de vitesse de propagation des ultrasons. Les vitesses ultrasonores ont été calculées à partir du temps de propagation de l'impulsion (précision $0.1 \mu \mathrm{s}$ ) et de la distance de propagation (précision de l'ordre du millimètre). La reconstitution de l'image tomographique a été réalisée en modélisant le parcours effectif de l'impulsion en fonction de la distribution des vitesses ultrasonores.

L'acquisition des données relatives aux temps de propagation a été réalisée in situ avec un appareil portable (AU 80 Sattec, France) équipé de deux transducteurs de fréquence $1 \mathrm{MHz}$. Dans d'autres recherches on avait travaillé à des fréquences plus faibles [9]. Dans ce cas on a choisi de travailler à $1 \mathrm{MHz}$ pour obtenir une meilleure résolution de l'image. Pour dépasser le problème de l'atténuation du signal, remarquable à cette fréquence, on a utilisé un transducteur source doué d'une énergie d'introduction très élevée et un transducteur de réception avec un dispositif d'amplification du signal reçu.

Dans le cas d'un signal trop entaché par le bruit, le signal a été enregistré et une analyse en fréquence a été faite pour choisir un filtre et ainsi éliminer une partie des harmoniques. Les fréquences de coupe ont été choisies en fonction des résultats de l'analyse spectrale et cela a servi finalement pour améliorer la lecture du temps de propagation des ultrasons.

Pour réaliser les images tomographiques, on a utilisé le logiciel Migratom $^{\circledR}[5,16]$, couramment employé dans le domaine de la géophysique. Les valeurs des temps de propagation et les coordonnées de chaque point de mesure ont été insérées dans le logiciel. Avant de commencer l'inversion tomographique, un traitement des données a été effectué pour démarrer correctement le logiciel : on a calculé la vitesse 
Tableau I. Valeurs des vitesses des ultrasons $(\mathrm{m} / \mathrm{s})$ mesurées sur le hêtre à trois niveaux.

\begin{tabular}{lcccccc}
\hline Section & $\begin{array}{c}\text { Niveau de la section } \\
\text { par rapport au sol }(\mathrm{m})\end{array}$ & $\begin{array}{c}\text { Périmètre de la section } \\
(\mathrm{m})\end{array}$ & $\begin{array}{c}\text { Valeurs moyennes } \\
(\mathrm{m} / \mathrm{s})\end{array}$ & $\begin{array}{c}\text { Écart type } \\
(\mathrm{m} / \mathrm{s})\end{array}$ & $\begin{array}{c}\text { Valeurs maximales } \\
(\mathrm{m} / \mathrm{s})\end{array}$ & $\begin{array}{c}\text { Valeurs minimales } \\
(\mathrm{m} / \mathrm{s})\end{array}$ \\
\hline $\mathrm{A}$ & 0.60 & 1.69 & 1180 & 160 & 1780 & 460 \\
$\mathrm{~B}$ & 0.84 & 1.59 & 1440 & 110 & 2350 & 680 \\
$\mathrm{C}$ & 1.38 & 1.38 & 1600 & 20 & 1910 & 1190 \\
\hline
\end{tabular}

moyenne, maximale et minimale dans la section, l'incertitude moyenne des temps de parcours, etc. La section du tronc a été virtuellement divisée en plusieurs cellules et à chaque cellule on a attribué une valeur de vitesse calculée par le logiciel. Le nombre et la taille des cellules ont été déterminés en tenant compte de différents facteurs tels que la taille de la section du tronc, le nombre des signaux enregistrés, la valeur du rayon de Fresnel. La taille des cellules représentait la taille minimale du défaut du bois qu'on pouvait détecter sur la section du tronc examinée.

Pour déterminer la distribution des vitesses dans la section, le logiciel utilisait un algorithme itératif, c'est-à-dire qui modifie la solution initiale (qui correspond à une hypothèse initiale sur la distribution des vitesses dans les cellules) jusqu'à convergence vers la solution cherchée. On a répété, pour chaque itération, le calcul du vecteur des temps - obtenu en modélisant la propagation de l'impulsion ultrasonore à l'intérieur de la section 2D hétérogène -, la comparaison entre les temps calculés et les temps mesurés et la correction des vecteurs des vitesses. Les itérations sont arrêtées lorsque l'on aboutit à une valeur préfixée de l'écart type entre les temps expérimentaux et les temps calculés ou quand toutes les itérations ont été élaborées.

Le résultat final est l'image tomographique de la section de l'arbre, où les zones correspondantes au bois non attaqué sont caractérisées par des valeurs de vitesse de propagation des ultrasons élevées, tandis que les zones dégradées sont représentées par de faibles valeurs de vitesse. Une relation similaire a été établie sur des éprouvettes attaquées au laboratoire par différents agents pathogènes $[1,3,18]$.

Les points de mesures par ultrasons ont été ensuite utilisés pour les mesures de la résistance à la pénétration de l' aiguille du « Résistograph».

\section{RÉSULTATS}

Les valeurs des vitesses mesurées sur le hêtre sont montrées dans le tableau I et sont comprises entre $460 \mathrm{~m} / \mathrm{s}$ et $2350 \mathrm{~m} / \mathrm{s}$. La reconstitution en 2D de l'image par tomographie ultrasonore de trois sections du tronc de l'arbre est montrée dans la figure 2. Sur la section A (Fig. 2f) on remarque que presque la moitié de la surface présente des valeurs inférieures à $1000 \mathrm{~m} / \mathrm{s}$, avec des zones de très faibles valeurs de $650 \mathrm{~m} / \mathrm{s}$. Apres l'abattage de l'arbre et l'analyse des sections, nous avons remarqué une progression de la dégradation du bois du bas vers le haut du tronc (Figs. 2c, 2e et 2f). Sur la section A, la distribution statistique des valeurs des vitesses mesurées (Fig. 3) présente une allure bi modale tandis que sur la section $\mathrm{C}$ on observe une distribution normale. Nous pouvons déduire que la section A est plus attaquée que la section $\mathrm{C}$.

La résolution de l'image tomographique calculée à partir des rayons de Fresnel est comprise entre 4 et $5 \mathrm{~cm}$, ce qui signifie que, pratiquement, les défauts de taille inférieure à cette dimension ne sont pas détectables sur l'image reconstituée.
La reconstruction de l'image tridimensionnelle à partir de trois tomogrammes en 2D de la distribution des vitesses (Fig. 4) a été faite par le logiciel Fortner. Cette image est très parlante, et elle montre l'évolution de la pourriture dans le tronc, de la base de l'arbre vers le haut.

La figure 5 confirme l'existence des zones de pourriture détectées avec le «Résistograph». La correspondance entre les valeurs des vitesses et la résistance à la pénétration de l'aiguille est montrée pour les points 0 et 4 de la section A. Aux valeurs faibles des vitesses correspondent des valeurs faibles de la résistance à la pénétration de l'aiguille. Nous remarquons que la quantité et la qualité des informations fournies par l'image tomographique est nettement supérieure à l'information fournie par le « Résistograph».

\section{DISCUSSION}

Nous avons vu que la technique de la tomographie ultrasonore proposée dans cet article a donné des résultats très satisfaisants pour la détection des défauts dans les arbres sur pied. En effet, avec cette technique il est possible de détecter les pourritures, de les localiser et de mesurer approximativement leur forme et leur étendue, en effectuant des mesures totalement non destructives. L'utilisation des ultrasons d'une fréquence relativement élevée de $1 \mathrm{MHz}$ permet d'avoir une bonne résolution de l'image (4-5 $\mathrm{cm}$ pour un diamètre du tronc de $60 \mathrm{~cm}$ ), résolution qui est certainement supérieure à celle qu'il est possible d'obtenir aujourd'hui avec des méthodes vibratoires dans le domaine acoustique (de fréquence inférieure à $20 \mathrm{kHz}$ ). Par ailleurs, nous pouvons dire que la méthodologie développée dans cet article représente une avancée très significative par rapport aux techniques actuellement utilisées, mais elle présente différents inconvénients comme :

- l'acquisition des données qui est relativement longue (45 min par arbre), mais cette limitation pourrait être améliorée à l'aide d'un appareil à ultrasons doué de plusieurs voies de mesure et d'un collier support pour plusieurs transducteurs pour permettre leur positionnement rapide autour de l'arbre ;

- la lecture des signaux qui peut parfois se révéler difficile mais peut toute fois être améliorée par une analyse en fréquence selon la procédure proposée par Martinis [9] et par l'utilisation de transducteurs spéciaux, ayant une grande énergie à l'émission. Une lecture automatique du signal, l'aide de la procédure nommée auto-picking, évitera les fautes de lecture dues à l'opérateur, en assurant l'acquisition rapide des données, pour les signaux avec un bon rapport signal/bruit. Pour les signaux très entaché par de bruit, il faudra une analyse fine, effectuée par un opérateur très spécialisé ; 


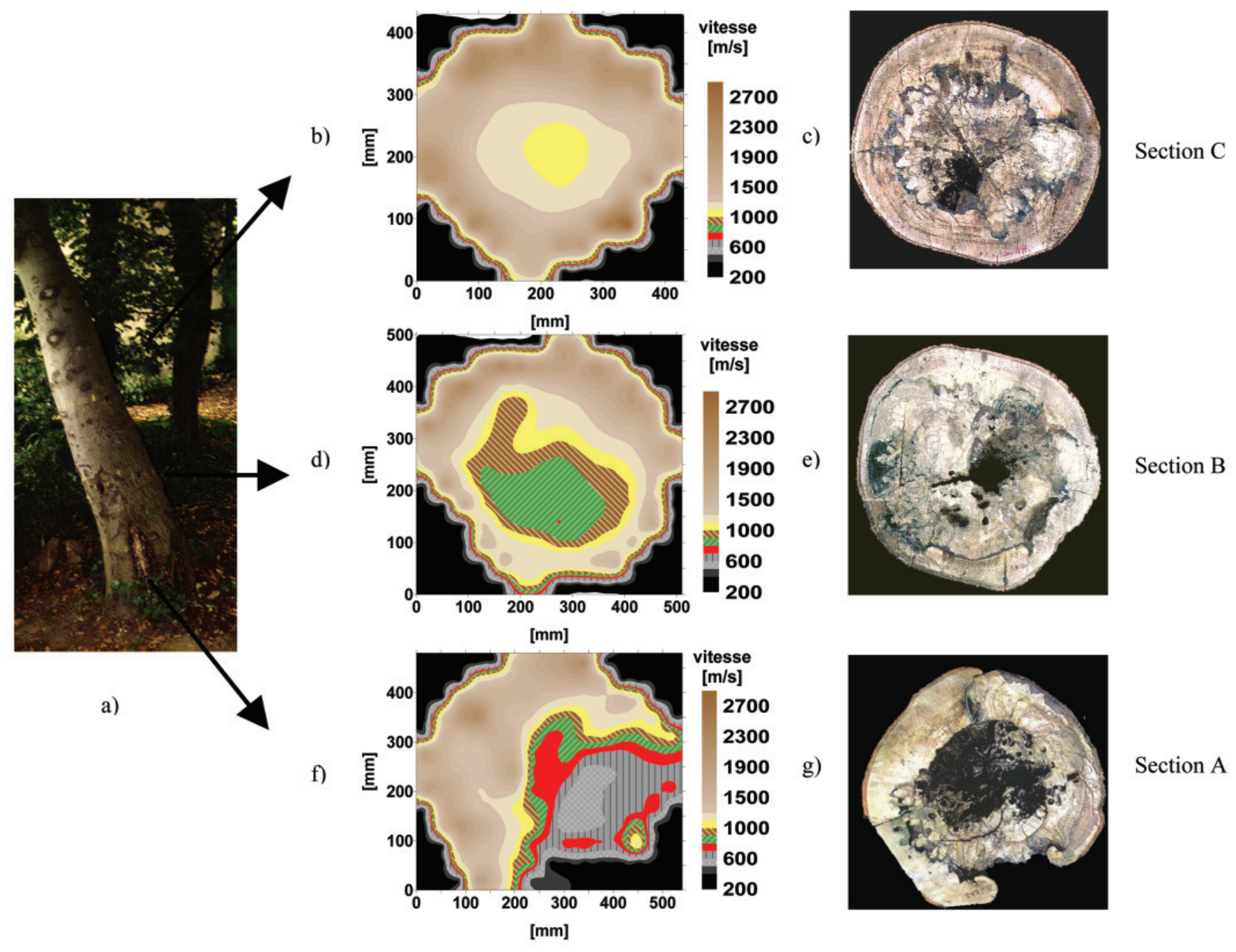

Figure 2. Images tomographiques obtenues à partir des vitesses de propagation des ultrasons dans le tronc d'un hêtre. Légende : (a) le tronc étudié ; (b), (d), (f) : images tomographiques correspondantes aux niveaux A $36 \mathrm{~cm} \mathrm{du} \mathrm{sol,} \mathrm{B} 84 \mathrm{~cm} \mathrm{du} \mathrm{sol,} \mathrm{C} 138 \mathrm{~cm}$ du sol ; (c), (e), (f) : images photographiques réelles de chaque section étudiée.

- l'analyse des données et l'utilisation du logiciel qui doivent être faites par un opérateur très qualifié, mais dans le futur il sera possible de développer un algorithme tomographique qui rendra la procédure automatique ;

- sur toutes les images reconstituées nous pouvons observer une couronne des vitesses très faibles, ce qui est probablement dû aux mesures faites à la périphérie du tronc et aux différences entre les vitesses mesurées selon la direction radiale et tangentielle ;

- la présence de petites cavités de 1 à $2 \mathrm{~cm}$ de diamètre sur la section du tronc est difficilement détectable. Ceci est dû à la résolution spatiale expérimentale $(4-5 \mathrm{~cm})$ et à la méthodologie tomographique employée qui distribue de forts contrastes de vitesses sur les cellules virtuelles de la surface étudiée et, par voie de conséquence, fait que les valeurs les plus grandes des vitesses sont sous-estimées tandis que les valeurs les plus faibles des vitesses sont surestimées. L'effet Wielandt pourrait expliquer ce phénomène, car les ondes de choc comme les impulsions ultrasonores évitent les zones de faibles vitesses [19] ;

- l'atténuation du signal ultrasonore dépend beaucoup du couplage transducteurs-écorce d'une part et d'autre part, de la présence éventuelle des zones avec de la pourriture. L'atténuation peut être utilisée comme paramètre pour diagnostiquer l'état de la structure interne de l'arbre seulement si on connaît le contraste d'impédance acoustique du couplage transducteurécorce pour tous les points de mesure ;

- l'analyse statistique de la résolution de la méthode à travers deux paramètres, le rayon de Fresnel et le nombre des mesures de temps de propagation de l'impulsion ultrasonore, confirme qu'il faut avoir un nombre suffisamment grand de mesures expérimentales faites à $1 \mathrm{MHz}$ pour obtenir des résultats satisfaisants du point de vue mathématique et physique, pour la reconstitution de l'image tomographique ultrasonore ;

- la grande anisotropie du matériau bois (grande différence entre les vitesses dans le plan transversal RT et les plans LR et 


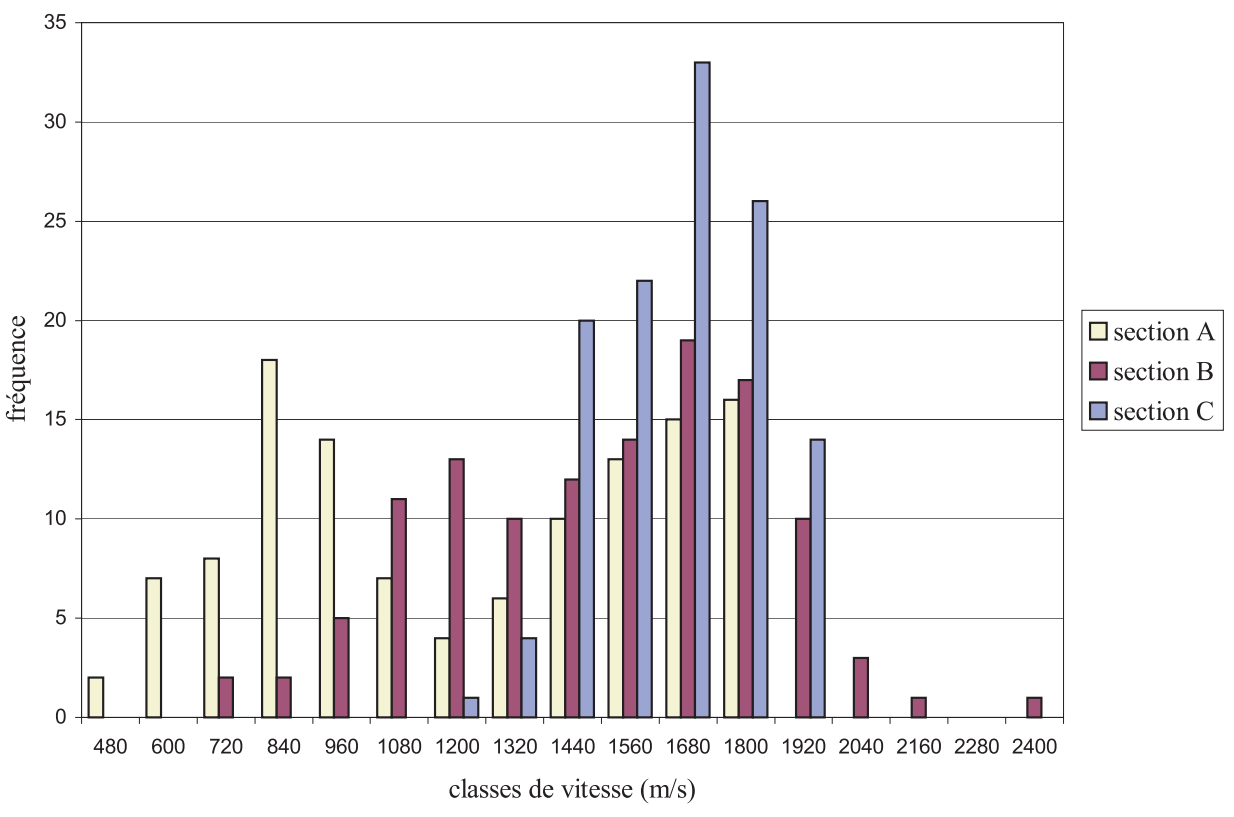

Figure 3. Distribution, pour chaque section du hêtre, de la fréquence des valeurs de vitesse par classes.

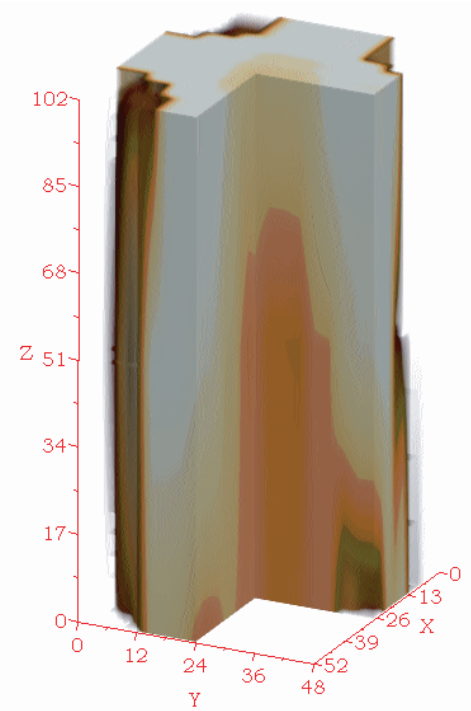

Figure 4. Image en 3D de la distribution des valeurs des vitesses dans le tronc d'arbre étudié.

LT) ne permet pas, à l'état actuel du développement technologique, de réaliser directement des tomographies en 3D. Pour obtenir une image en 3D on doit effectuer au minimum trois tomographies en 2D afin de visualiser le volume de la pourriture dans le tronc de l'arbre.

\section{CONCLUSION}

La grande efficacité de la tomographie ultrasonore, avec sa capacité de détecter, localiser et mesurer les zones dégradées par mesures non destructives nous autorise de proposer cette technique comme instrument pour diagnostiquer la présence dangereuse des pourritures sur les arbres sur pied des parcs et

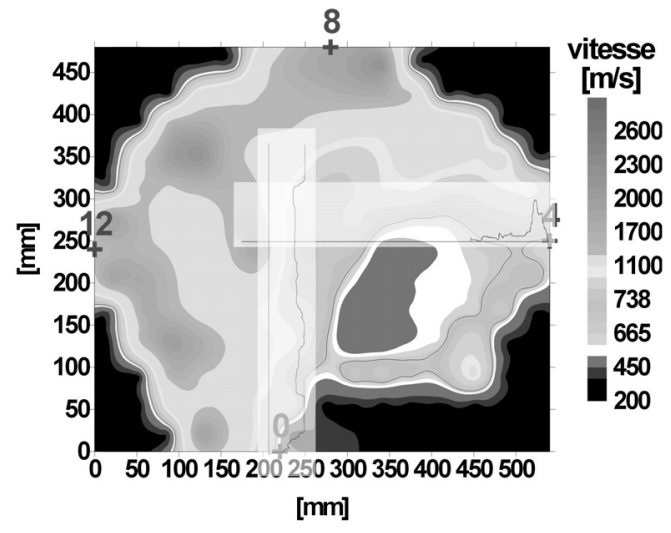

Figure 5. Superposition du profil de la résistance à la pénétration de l'aiguille du Résistograph sur l'image tomographique de la section A points 0 et 4 .

jardins publiques. Dans le futur, cette technique pourrait devenir un instrument de travail quotidien pour la gestion de l'espace vert urbain.

\section{RÉFÉRENCES}

[1] Bauer C., Kilbertus G., Bucur V., Technique ultrasonore de caractérisation du degré d'altération des bois de hêtre et de pin soumis à l'attaque de différents champignons, Holzforschung 45 (1991) 41-46.

[2] Biagi E., Gatteschi G., Masotti L., Zanini A., Tomografia ad ultrasuoni per la caratterizzazione difettologica del legno, Alta frequenza - Rivista di elettronica 6 (1994) 48-57.

[3] Bucur V., Acoustics of Wood, CRC Press Inc., 1995.

[4] Davis J.R., Lerdin A., Wells P., Ilic J., X-ray microtomography of wood, J. Inst. Wood Sci. 12 (1991) 259-261. 
[5] Dines K., Litle J., Computerized Geophysical Tomography, Proc. IEEE, 67, 1979, pp. 1065-1073.

[6] Divos F., Stress wave based tomography for tree evaluation, in: Proc. of 12th Int. Symposium on NDT of Wood, Sopron 13-15 Sept. 2000, p. 469.

[7] Habermehl A., Ridder H. W., Seidl P., Computerized Tomographic Systems as tools for diagnosing urban tree health, Proc. Int. Symp. on Urban Tree Health, Acta Hortic. 496 (1999) 261-268.

[8] Link R., Nuding W., Sauerwein K., Wiacker H., Habermehl A. Ridder H.W., Mobile Computerized Tomographic Systems: MCT 3 and MCT 5, Giornale delle prove non distruttive, 2 (1987) 25-27.

[9] Martinis R., Analisi e sviluppo di tecniche non invasive per la valutazione di carie in alberi in piedi, Thèse de Doctorat, Dipartimento di Biotecnologie Agrarie, Facoltà di Agraria, Firenze, 2002.

[10] Pearce R.B., Fisher B.J., Carpenter T.A., Hall L.D., Water distribution in fungal lesions in the wood of sycamore (Acer pseudoplata$n u s)$, determined gravimetrically and using nuclear magnetic resonance imaging, New Phytol. 135 (1997) 675-688.

[11] Rayner A.D.M., Boddy L., Fungal decomposition of wood, Its biology and ecology, John Wiley \& Sons Ltd., 1988.

[12] Ross R.R., Using sound to evaluate standing timber, Int. For. Rev. 1 (1999) 43-44.
[13] Rust S., Göcke L., PICUS Sonic Tomograph - a new device for nondestructive timber testing, in: International Symposium on Plant Health in Urban Horticulture, Braunschweig, Germany, 2000, p. 300.

[14] Schad K.C., Kretschmann D.E., McDonald K.A., Ross J.R., Green D.W., Stress Wave Techniques for Determining Quality of Dimensional Lumber From Switch Ties, FPL-RN-0265 USDA, Forest Products Laboratory, 1995.

[15] Tomikawa Y., Iwase Y., Arita K., Yamada H., Nondestructive inspection of wooden poles using ultrasonic computed tomography, IEEE Transaction on UFFC, 33 (1990) 354-358.

[16] Tweeton D.R., A Tomographic Computer Program With Constraints To Improve Reconstructions for Monitoring In Situ Mining Leachate, BuMines RI 9411, 1988.

[17] Wang X., Ross R.J., McClellan M., Barbour R.J., Erickson J.R., Forsman J.W., McGinnis G.D., Strength and stiffness assessment of standing trees using a nondestructive stress wave technique, USDA, Forest Service; FPL - RP - 585, 2000

[18] Wilcox W.W., Detection of early stages of wood decay with ultrasonic pulse velocity, For. Prod. J. 38 (1988) 68-73.

[19] Wielandt E., On the Validity of Ray Approximation for Interpreting Delay Times, in: Wielandt E., Seismic Tomography, G. Nolet, Reidel, 1987, pp. 85-98. 